\title{
Curcuma Longa Against Nitromethanesulfonamide Induced Hepatic Injury In A Rat Model.
}

\author{
Abroo Fatima Qazi ${ }^{1}$,Din Muhammed Shaikh ${ }^{2}$ \\ ${ }^{I}$ (Department Of Physiology Faculty Of Medicine \& Allied Medical Sciences, ISRA University, Hyderabad, \\ Pakistan) \\ ${ }^{2}$ (Department Of Physiology, Faculty Of Medicine \& Allied Medical Sciences, ISRA University, Hyderabad, \\ Pakistan)
}

\begin{abstract}
Curcuma Longa has been used in traditional medicine as a house hold remedy for various diseases. This experimental case control study was conducted to observe the protective effect of Curcuma Longa in NitroMethanesulfonamide induced hepatotoxicity in the animal house at ISRA university Hyderabad.40 male albino Wistar rats were equally divided into four groups of 10 each. Group A used as the control group and was fed the normal rat chow for 09 days. Group B was fed with Nitro-Methane sulfonamide for 09 days followed by Curcuma Longa for next 09 days. Group $C$ was given Curcuma Longa for 09 days followed by NitroMethanesulfonamide for next 09 days. Group $D$ was given Nitro-Methanesulfonamide in combination with Curcuma Longa for 09 days. After 24 hours of feeding, blood samples from groups A and D were taken from dorsal vein in the tail of rats for biochemical analysis of serum levels of ALT, AST, ALP and GGT at days 01 and $09 \&$ for groups $B$ and $C$ blood samples were collected at day 01, day 09 and day 18.The analysis showed that prevention from the Nitro-Methane sulfonamide induced hepatotoxicity by the Curcuma Longa administration was observed after 18 days with a $p$ value $<0.012$, and Pearson's correlation for regression at 0.06 and -0.07.The results have clearly proven that Curcuma Longa acts as a hepatoprotective agent against "over the counter" drug induced hepatic injuries.
\end{abstract}

Keywords : Curcuma longa, Hepatotoxicity, Nitromethanesulfonamide, Traditional medicine,NSAIDs

\section{Introduction}

Curcuma Longa has been used in traditional medicine as a house hold remedy for various diseases including biliary disorders, anorexia, cough, diabetic wounds, hepatic disorders, rheumatism and sinusitis. It is reported to have anti-inflammatory, anti-oxidant, anti-viral, anti-ulcer, anti-carcinogenic and hypocholesteremic activities [1][2]. Curcuma Longa (Turmeric) a perennial herb from Zingiberaceae family is cultivated in China, India and Asia [3]. Diferuloylmethane (Curcumin) has been isolated as the active ingredient of Curcuma longa. Curcumin is reported to have anti-viral, anti-fungal, anti-inflammatory, hepatoprotective and anti-oxidant properties [4][5].

Nitromethanesulfonamide (NMS) is a selective cyclooxygenase II inhibitor non-steroidal antiinflammatory drug (NSAID) and it is being marketed with the generic name of nimesulide [6]. NSAIDS are the drugs that inhibit the production of prostaglandins (PGs) through inhibition of COX-1 and COX-2, the enzymes responsible for the synthesis of PGs [7]. For NSAIDS, the most common adverse events are the gastrointestinal irritation because of this their use is under scrutiny. NMS is widely used NSAID that differs from other NSAIDS in structure and mode of action, claiming fewer gastrointestinal side effects due to its preference for cox-2 enzyme [8]. But it is reported to cause hepatotoxicity by elevating the hepatocellular enzymes [9].

In many Asian countries, the use of Curcuma Longa as a food spice, colorant and medicine has a long tradition. It is extensively used orally and topically in traditional Chinese and Indian systems of medicine for a wide range of indications including abdominal fullness, kidney pain, amenorrhea and arthritis. In both the Ayurveda and Siddha systems of medicine, a turmeric paste is applied topically to treat ulcers and scabies [10]. Curcumin protects cells against lipid peroxidation. This may be due to the anti-oxidative effects of the phenolic groups of curcumin [10]. Curcumin is reported to be an effective agent in decreasing serum aspartate transaminase and alkaline phosphatase activity, and free fatty acid, cholesterol and phospholipid levels [11].There is a decreased prevalence of hepatic toxicity and injury in Pakistan as compared to that seen and reported in the western countries on administration of over the counter (OTC) potentially toxic drugs that are imbibed by the consumers with or even without prescription of the registered medical practioners.

For this purpose, we wanted to determine the role of spices (Garam Masala) that are used in almost all form of cuisine prepared in our country. Special emphasis was given to Curcuma Longa because this alone is present in almost all dishes eaten in all strata of socio-economic status alike. 


\section{Material and methods}

2.1 Ethical Approval: the protocol of experiment was approved by the Institutional Ethical Committee of ISRA University, Hyderabad.

2.2 Experimental animals: Total 40 healthy male Wistar rats weighing $150 \mathrm{gms}$ to $200 \mathrm{gms}$ were used in the current study. All animals were acclimatized to animal house's condition for fortnight before commencement of the experiment. The animals were divided into 4 groups (10 in each) and housed in separate cages at a controlled temperature of $18-26^{\circ} \mathrm{C} / 64-78^{\circ} \mathrm{F}$ and a constant light-dark schedule ( 10 hours' light and 14 hours' dark cycle). Animals were fed with diet that was formulated according to the guidelines provided by the Institute for Laboratory Animal Research and mineral water was given ad libitum as specified in the journal of Institute of Laboratory Animal Resources in 2011.

2.3 Chemicals: Nimesulide was purchased from local pharmacist. Dried Rhizomes of Curcuma Longa were obtained from the local market.

\subsection{Preparation of Curcuma Longa Powder:}

The dried rhizomes were completely desiccated and to insure absence of atmospheric moisture, the rhizomes were dehydrated in a hot air oven at $70^{\circ} \mathrm{C}$ for 06 hours. Thereafter, they were ground in a high-speed grinder to get a fine powder that was kept in an airtight jar until used. follows:

2.5 Data collection procedure: The animals were equally divided into four groups of 10 each as

Group A: used as the control group and was fed the normal diet for 09 days.

Group B: was fed with Nitro-Methanesulfonamide for 09 days followed by Curcuma longa for next 09 days. Group C: was pretreated with Curcuma Longa for 09 days followed by Nitro-Methanesulfonamide for next 09 days.

Group D: were given Nitro-Methanesulfonamide in combination with Curcuma longa for 09 days.

After 24 hours of feeding, Blood samples from groups A \& D were taken from dorsal tail vein for biochemical analysis of serum levels of ALT, AST, Alkaline Phosphatase and $\mathrm{Y}$-GT through automated analyzer at clinical laboratory, ISRA University Hyderabad, at days 0 and 09 . For groups B \& C blood samples were collected at day 0 , day 09 and day 18 .

\section{Results and Discussion}

The current study was carried to illuminate the hepatoprotective and anti-oxidative properties of Curcuma Longa, when taken orally; alone as a homeostatic booster and in combination with NitroMethanesulfonamide (NMS) (Which is a known hepatotoxic agent) to determine the curative properties of Curcuma Longa following hepatic injury.

Table I: Hepatic Enzyme levels (IU/L) for Control shown as Math. Mean.

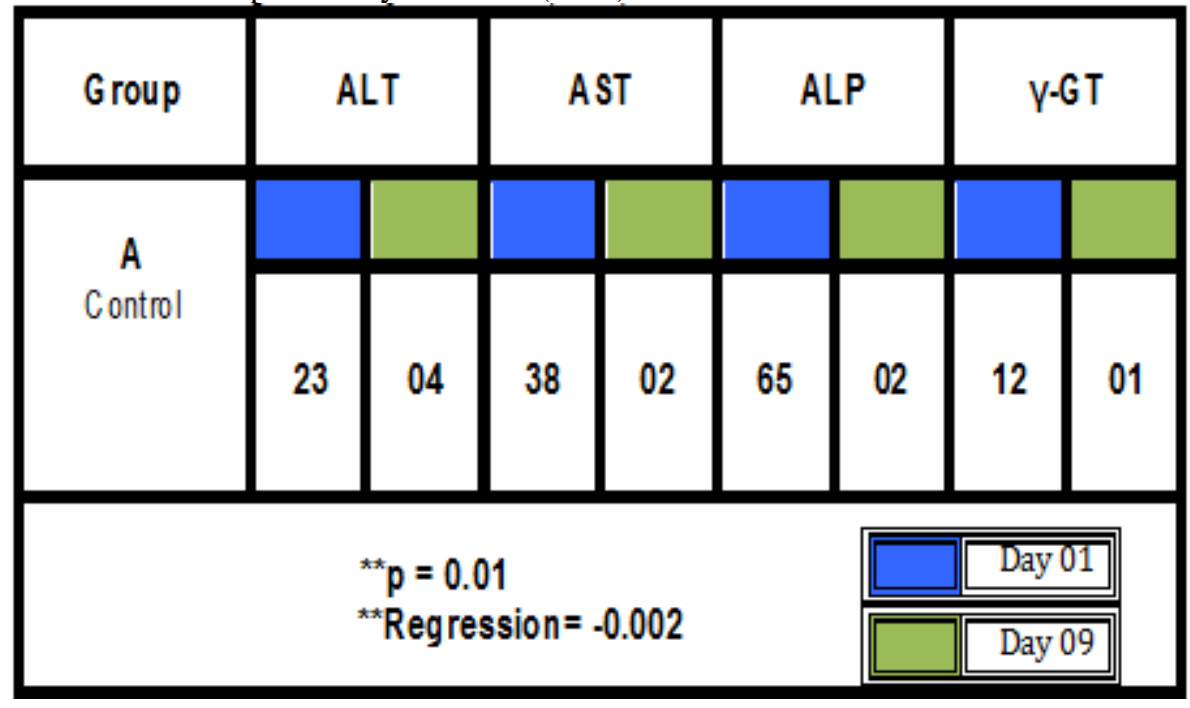

Table: 01 clearly shows the four groups in which ALT was done. The control group did not receive either substances and gave us the normal value for this enzyme. The group B received N-MS for nine days and it is clearly visible that the mean ALT value in this group was $168 \mathrm{IU} / \mathrm{L}$ on 9th day which decreased to $40 \mathrm{IU} / \mathrm{L}$ on $18^{\text {th }}$ day following nine days Curcuma longa administration. In Table: II, the same trend is visible for AST, Alkaline Phosphatase and $\mathrm{Y}$-GT. 
Table II: Hepatic Enzyme levels (IU/L) for Test Group B shown In Math. Mean.

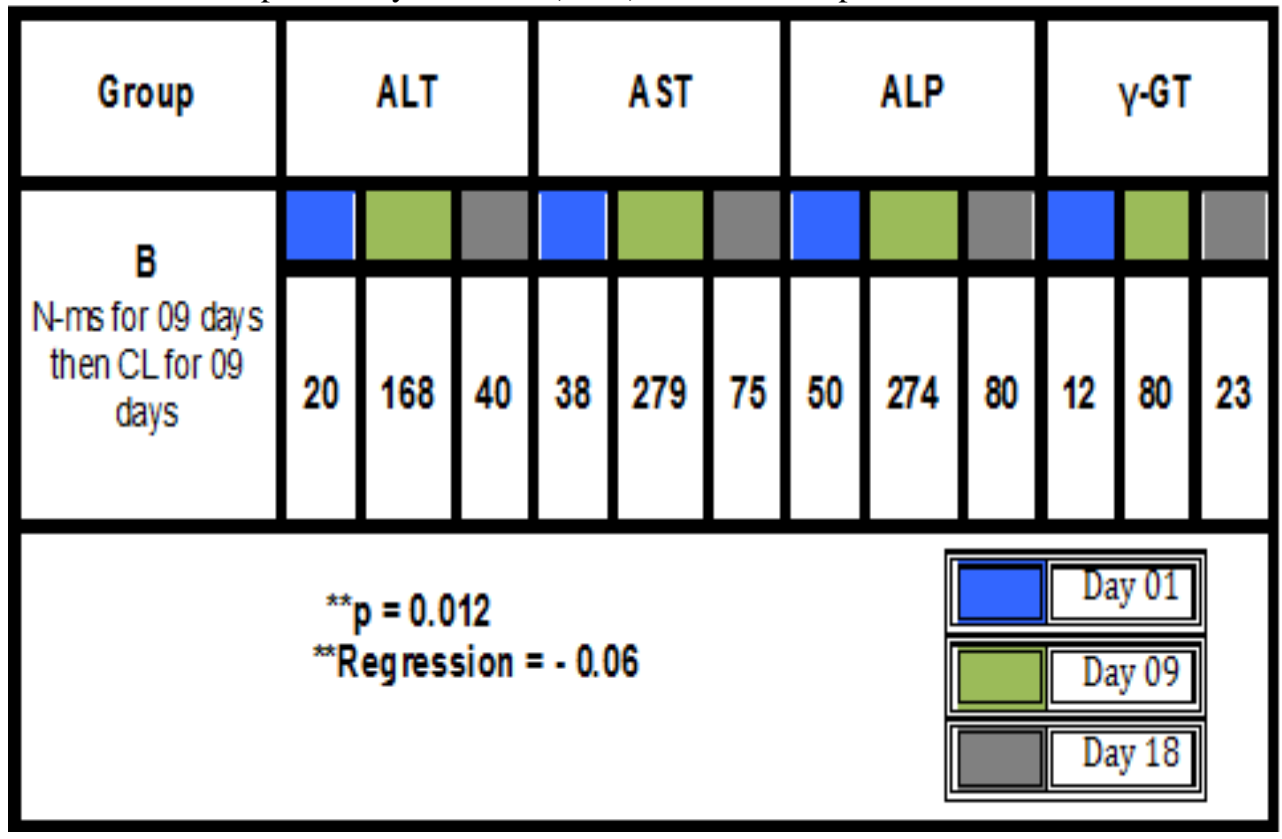

Group C received Curcuma longa for nine days to assess any toxic effect of this natural herb on LFTs and also to find out whether Curcuma Longa will or will not boost the anti-oxidative capabilities of hepatic antioxidative systems. The results were self-explanatory that on day 09, the LFTs showed mean value of less than the normal control, thereby alleviating the fears of Curcuma Longa induced hepatotoxicity as seen in Table: III.

However, much to our satisfaction, the results of day 18 of all these enzymes as compare to the same on day 09 of Group B shows a remarkable decrease in the hepatotoxic potentials of N-MS when given following prior Curcuma longa use.

Table III: Hepatic Enzyme levels (IU/L) for Test Group B shown In Math. Mean.



We also wanted to check the homeostasis boosting capability of Curcuma longa when given as the time as N-MS which was done in Group D. The results are self-explanatory that on day 09 of group D as compared to day 09 of Group B showed a very convincing argument in favor of Curcuma Long as shown in Table: IV. 
Table IV: Hepatic Enzyme levels (IU/L) for Test Group D shown In Math. Mean

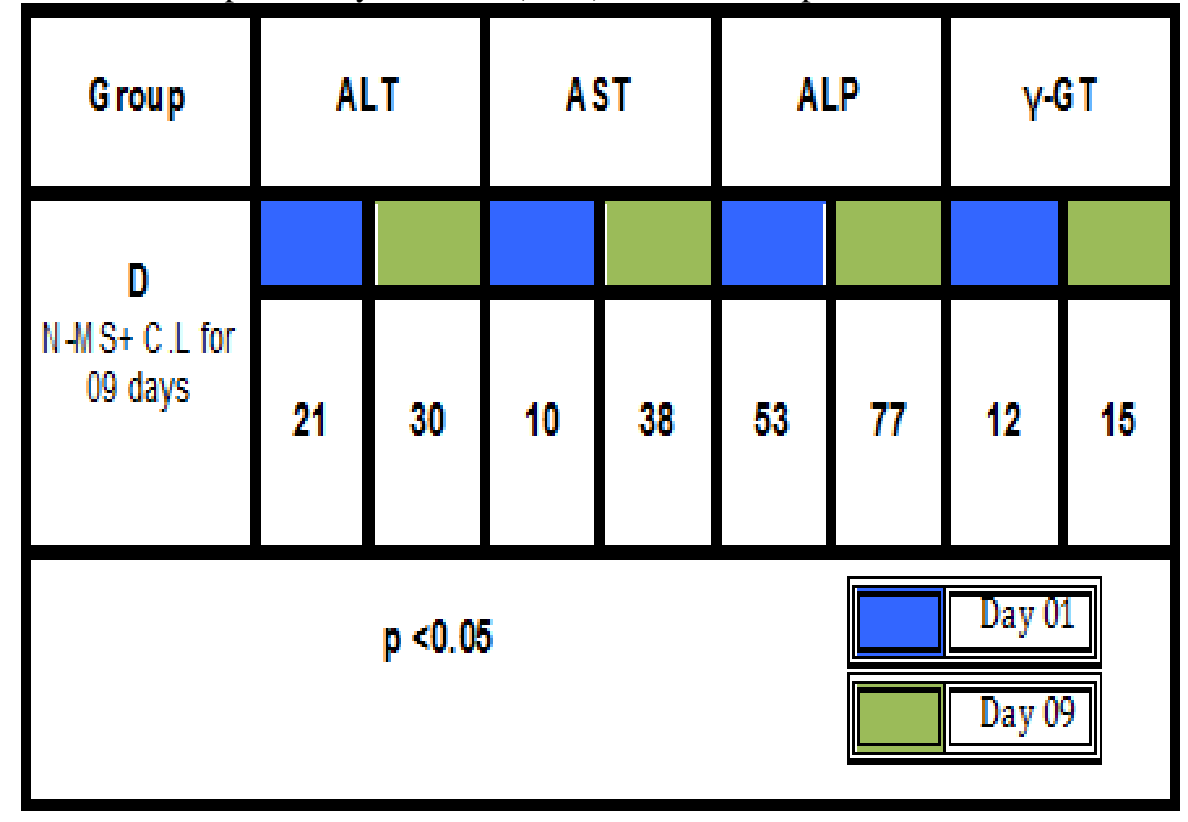

IV. Conclusion

The present study implies that the curcuma longa has a protective effect on hepatic injury induced by NMS.

\section{References}

[1]. Oguzturk, H., Ciftci, O., Aydin, M., Timurkaan, N., Beytur, A. \& Yilmaz. Ameliorative effects of curcumin against acute cadmium toxicity on male reproductive system in rats. International Journal of Andrology 2011; 44, 243-249.

[2]. Nonose, N., Pereira, J.A., Machado, P.R.M., Rodrigues, M.R., Sato, D.T. and Martinez, C.A.R. Oral administration of curcumin (curcuma longa) can attenuate the neutrophil inflammatory response in zymosan-induced arthritis in rats. Acta Cirurgica Brasileira 2014; 29(11), pp.727-734.

[3]. Li, G., Chen, J.-B., Wang, C., Xu, Z., Nie, H., Qin, X.-Y., Chen, X.-M. \& Gong, Q. 2013. Curcumin protects against acetaminophen-induced apoptosis in hepatic injury. World Journal of Gastroenterology 2013;19, 7440-7446.

[4]. Nawaz A, Khan Gm, Hussain A, Ahmed A, Khan A and Safdar M. Curcumin: a natural product of biological importance. Journal of Research 2011; vol: 27(1); pp 7-14

[5]. Singh Bk, Tripathi M, Chaudhari Bp, Pandey Pk, Kakkar P. Natural terpenes prevent mitochondrial dysfunction, oxidative stress and release of apoptotic proteins during nimesulide-hepatotoxicity in rats. Plos one 2012; vol: 7(4): e34200.

[6]. Zanwar, A.A., Badole, S.1. and Menaa, F. Curcuma longa: use for skin disease care. In bioactive dietary factors and plant extracts in dermatology 2013; (pp. 391-396). Humana press.

[7]. Kress, H.G., Baltov, A., Basiński, A., Berghea, F., Castellsague, J., Codreanu, C., Copaciu, E., Giamberardino, M.A., Hakl, M., Hrazdira, 1. And Kokavec, M. Acute pain: a multifaceted challenge-the role of nimesulide. Current Medical Research and Opinion 2016; 32(1), pp.23-36.

[8]. Minhaj, M.A. and Alam, M.S., Pharmacological evaluation of berberine against nimesulide induced hepatoxicity. International Journal of Scientific Research 2014, 3(5), pp 798-802.

[9]. Nwozo, S.O., Osunmadewa, D.A. and Oyinloye. Anti-fatty liver effects of oils from zingiber officinale and curcuma longa on ethanol-induced fatty liver in rats. Journal of Integrative Medicine 2014; 12(1), pp.59-65.

[10]. Kumar, a. A review on hepatoprotective herbal drugs. International Journal of Research in pharmacy \& chemistry 2012; $2,92-102$.

[11]. Saraswati, T.R, Manalu. W, E kastuti.D.R, and Kusumorini. N. The role of turmeric powder in lipid metabolism and its effects on quality of the first quail's egg. Journal of The Indonesian Tropical Animal Agriculture 2013; 38(2), p 123-130. 